\title{
Crepidula fornicata L. (mollusque, gastéropode) en baie de Marennes-Oléron : cartographie des fonds par sonar à balayage latéral et estimation du stock
}

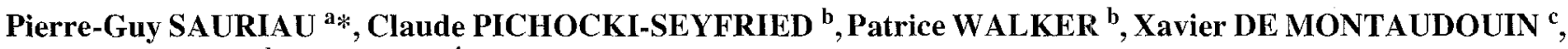 \\ Christian PALUD ${ }^{b}$, Maurice HÉRAL ${ }^{\text {a }}$ \\ ${ }^{a}$ CREMA (CNRS-IFREMER), place du Séminaire, BP 5, 17137 L'Houmeau, France \\ ${ }^{\mathrm{b}}$ CREOCEAN, 23 allée des Tamaris, 17000 La Rochelle, France \\ ${ }^{c}$ Laboratoire d'océanographie biologique, Université Bordeaux 1, URA 197, 2 rue du Professeur Jolyet, 33120 Arcachon, \\ France
}

(Revised 28/11/97, accepted 12/12/97)

\begin{abstract}
Crepidula fornicata L. (Mollusca, Gastropoda) in the Marennes-Oléron Bay: side-scan sonar mapping of subtidal beds and stock assessment. Extensive and detailed subtidal ground mapping of the Marennes-Oléron Bay and Fouras-Aix areas was performed with a side-scan sonar. Side-scan sonographs gave a comprehensive view of sedimentological bottom environments and also allowed us to outline locations of grounds colonised by the gastropod mollusc Crepidula fornicata $\mathrm{L}$. with a precise estimate of their surface. A sedimentological map of the centre of the Marennes-Oléron Bay is given, describing spatial organisation of the following four types of sediment: pure mud, fine sand, coarse sand and rocks. Crepidula beds were also recognised on sonographs and were estimated to cover 181 hectares in the Fouras-Aix area and 615 hectares within the Marennes-Oléron Bay. Standard sampling methods were then combined with the side-scan sonar mapping results, allowing an estimate to be made of the stocks of Crepidula fornicata (live specimens and dead shells). A stratified sampling procedure was performed in April-May 1995, including 80 stations sampled with a Smith McIntyre grab (two grabs per station). The live stock of Crepidula was estimated to be $2494 \pm 3344$ tons at Fouras-Aix and $2644 \pm 1137$ tons within the bay of Marennes-Oléron. This latter estimate is similar to the previous one performed in this bay in spring 1984, i.e. $1800 \pm 900$ tons, due to the overlap of $95 \%$ confidence intervals. These comparable estimates within the Marennes-Oléron Bay give no evidence to suggest that dredging operations (1 000-1500 tons $\left.\cdot \mathrm{yr}^{-1}\right)$ performed in the bay for 15 years are not efficient. However, annual landing operations of slipper limpet cannot prevent further spread of the species, as pointed out by the large development of a new Crepidula ground in the north-western centre of the bay (north Lamouroux sand bank). Both natural processes (current action and bed load transport) and bottom-trawl activities can facilitate spread of the species in the bay, as revealed by side-scan sonar image analysis. (C) Elsevier, Paris
\end{abstract}

\section{Crepidula fornicata / mapping / stock assessment / side-scan sonar / Marennes-Oléron Bay}

Résumé - Une reconnaissance exhaustive de la zone subtidale de la baie de Marennes-Oléron et de la presqu'île de Fouras a été réalisée à l'aide d'un sonar à balayage latéral. Ce levé a permis d'établir, avec une précision métrique, une cartographie continue des principaux faciès sédimentaires et un recensement, quasi complet, des populations de la crépidule Crepidula fornicata L. (mollusque, gastéropode). Quatre faciès sédimentaires sont individualisés sur les sonogrammes : vase, sable fin, sable gravier et roche. De même, la présence de crépidules organisées en tapis, en tâches ou bien en chaines éparses, est reconnaissable. L'analyse des enregistrements sonar a permis, après validation par prélèvements ponctuels,

* Correspondence and reprints 
d'individualiser 18 gisements de crépidules. Ils couvrent 181 hectares sur la presqu'île de Fouras-d'île d'Aix et 615 hectares en baie de Marennes-Oléron.

Afin d'en estimer le stock, la cartographie des gisements a été couplée à un échantillonnage quantitatif à la benne Smith Mc-Intyre selon un plan aléatoire stratifié de 80 stations. Le stock de crépidules vivantes fut estimé en avril-mai 1995 à $2494 \pm 3344$ tonnes sur le secteur Fouras-Ile d'Aix et à $2644 \pm 1137$ tonnes en baie de Marennes-Oléron. Cette dernière estimation est considérée comme équivalente à celle de 1984 en baie de Marennes-Oléron, soit $1800 \pm 900$ tonnes. Cette comparaison laisse supposer, en regard de la situation des gisements de crépidules non régulés de Fouras ou de Bretagne nord, que les dragages de nettoyage, organisés annuellement depuis 1980 en baie de Marennes-Oléron, s'avèrent globalement efficaces pour limiter la prolifération de cette espèce. Cependant, ces opérations de nettoyage n'empêchent pas la dispersion progressive de chaînes de crépidules du centre vers le nord de la baie (nord-ouest du banc de Lamouroux), que cette dispersion soit naturelle ou bien facilitée par les activités de chalutage démersal. (C) Elsevier, Paris

\section{Crepidula fornicata / cartographie / stock / sonar à balayage latéral / Marennes-Oléron}

\section{INTRODUCTION}

Répertorié en baie de Bourgneuf dès 1964 [25] au cours de son expansion le long du littoral Manche-Atlantique [4], le mollusque gastéropode Crepidula fornicata L. est apparu en 1969-1970 en baie de Marennes-Oléron [23, 10] ainsi que sur la presqu'île de Fouras [21]. En une dizaine d'années, il confirma sa réputation de "peste ostréicole "précédemment décrite dans les centres conchylicoles de Grande-Bretagne [7, 27, 6], des Pays-Bas [20] et de Bretagne [24]. Le développement d'un banc compact de crépidules a localement pour conséquences, outre la création d'entraves à la navigation, un envasement et un exhaussement accéléré des fonds, ce qui rend ces derniers impropres à la culture de l'huître [33]. Cette compétition pour l'espace se double d'une compétition trophique avec les cheptels ostréicoles [2, 11]. En termes de gestion et d'aménagement des écosystèmes conchylicoles, cela nécessite l'estimation respective de leurs stocks et productions $[11,17]$ préalablement à la mise en place d'opération de nettoyage des fonds à crépidules avec rejet à terre, traitement et rejet en mer et/ou commercialisation de produits dérivés.

En baie de Marennes-Oléron, une première cartographie descriptive des bancs de crépidules a été réalisée en 1981 à partir d'un échantillonnage ponctuel de 110 stations à la drague Charcot et 24 stations à la benne Orange-Peel en bordure des chenaux [26]. Une estimation quantitative des stocks de l'ensemble des mollusques compétiteurs trophiques des huîtres de la baie fut ensuite entreprise au printemps 1984. Cette estimation basée sur un échantillonnage aléatoire stratifié comportait 370 stations échantillonnées à la benne Smith Mc-Intyre [30]. L'estimation du stock de crépidules vivantes fut de $1800 \pm 900$ tonnes avec une précision de $50 \%$. Ce stock se répartis- sait, comme en 1981, préférentiellement au centre de la baie [31]. Cependant, une telle stratégie d'échantillonnage, bien que lourde et optimisée, ne peut permettre d'estimer précisément, pour une seule espèce comme la crépidule, à la fois la localisation géographique des bancs, leurs stocks et la modification temporelle de leur répartition spatiale.

Ie présent travail s'inscrit dans cette démarche et décrit la mise en place, à l'échelle de la presqu'île de Fouras, de l'île d'Aix et de la baie de Marennes-Oléron, de deux protocoles successifs afin de réactualiser l'état de la prolifération de la crépidule et d'en estimer le stock. Le premier protocole est calqué sur les travaux de cartographie biosédimentaire des fonds marins développés en baie de Saint-Brieuc par Hamon et Blanchard [14] et Hamon [15] : la description des faciès bio-sédimentaires est basée sur l'imagerie acquise par sonar à balayage latéral. L'interprétation bio-sédimentaire des images acoustiques est ensuite validée par l'analyse biologique et sédimentologique de prélèvements ponctuels judicieusement localisés. Le second protocole consiste en un échantillonnage quantitatif de chacun des gisements de crépidules préalablement individualisés el s'appuie sur les estimations validées de leur superficie. Le résultat global de cet échantillonnage stratifié doit permettre, à court terme, d'améliorer les mesures de gestion des populations de crépidules engagées depuis quinze ans en baie de MarennesOléron visant à réduire la prolifération de cette espèce.

\section{MATÉRIEL ET MÉTHODES}

\subsection{Sonar à balayage latéral}

Cet outil, utilisé depuis plus de vingt ans en géophysique, essentiellement en matière de travaux sous-marins ou de 
cartographie des champs pétroliers [28], est aussi employé à des fins de reconnaissance de la nature sédimentologique des fonds marins [1]. Il permet parallèlement d'étudier la dynamique sédimentaire $[16,3]$ et a été utilisé, plus récemment, en cartographie bio-sédimentaire $[14,15]$. Le sonar à balayage latéral employé est de type Dowty Widescan II. Il est constitué par un poisson de faible encombrement $(1.50 \mathrm{~m}$ de long pour $15 \mathrm{~kg})$, remorqué derrière le navire par un câble électroporteur qui transmet l'information à une unité de traitement du signal. Les ondes de haute fréquence (100 à $315 \mathrm{kHz})$, d'impulsion courte $(0,3 \mathrm{~ms})$ sont émises à une cadence très rapide (100 à $250 \mathrm{~ms}$ ) et avec une grande directivité garantissant des informations de haute résolution spatiotemporelle. La succession rapide des tirs permet d'obtenir des informations en continu traduisant la nature des fonds et leur morphologie sur une bande de 100 à $400 \mathrm{~m}$ de largeur, avec une résolution de l'ordre de quelques dizaines de centimètres. Entre août et novembre 1994, $1300 \mathrm{~km}$ environ de profils parallèles et jointifs ont été réalisés par temps calme et en dehors des périodes de grande marée, pour couvrir l'ensemble des $90 \mathrm{~km}^{2}$ de zones subtidales de la baie de Marennes-Oléron (entre Fort Boyard et l'embouchure de la Charente au nord, l'embouchure de la Seudre et le Pertuis de Maumusson au sud) et des secteurs subtidaux de Fouras-Ile d'Aix.

\subsection{Positionnement géographique par G.P.S. (Global Positioning System)}

Le corollaire indispensable à la mise en oeuvre des techniques d'imagerie par sonar et leur validation par prélèvements ponctuels est de pouvoir disposer d'un système de positionnement de précision suffisante pour garantir à la fois la bonne juxtaposition des bandes images (sonogrammcs) ainsi quc l'échantillonnage de zones d'une dizaine de mètres carrés. Cette précision a été obtenue en utilisant un récepteur des signaux GPS (NR 51 de SERCEL) fonctionnant en mode différentiel avec une précision à moins de $5 \mathrm{~m}$ près, pendant $95 \%$ du temps.

\section{3. Échantillonnage ponctuel pour la validation bio- sédimentaire}

La validation des faciès acoustiques a été effectuée le 13 septembre 1994 et le 7 mars 1995 par des prélèvements sédimentaires à la benne Shipeck $\left(0,04 \mathrm{~m}^{2}\right)$. Quatre faciès sont ainsi individualisés : vase, sable fin, sable- gravier et roche (voir [3] pour une discussion sur les limites de la méthode).

La présence ou l'absence suspectée de crépidule sur les images sonar a été validée par des prélèvements à la drague à pétoncles (maille de $35 \mathrm{~mm}$ ) traînée sur 100 à $200 \mathrm{~m}$. Cette validation a permis de circonscrire 18 gisements de crépidules dont la superficie est connue précisément.

\section{4. Échantillonnage ponctuel pour l'estimation du stock de crépidules}

L'estimation du stock de crépidules de ces 18 gisements a été réalisée à partir d'un échantillonnage aléatoire stratifié (voir [5] pour le détail des calculs des moyennes et variances) avec toujours un minimum de deux stations par strate. L'effort d'échantillonnage de 80 stations fut proportionnel à la superficie des bancs. Ce protocole, conduit du 18 au 21 avril et du 2 au 3 mai 1995, a consisté en des prélèvements dupliqués à la benne Smith Mc-Intyre $\left(0,10 \mathrm{~m}^{2}\right)$. Les échantillons ont été tamisés sur maille carrée de $1 \mathrm{~mm}$ et la faune benthique traitée selon les protocoles cités par Sauriau [30].

Les estimations de stock total ainsi que leurs intervalles de confiance à $95 \%$ sont calculés selon les formulations de Cochran [5].

\section{RÉSULTATS}

\subsection{Interprétation des sonogrammes}

\subsubsection{Faciès sédimentologiques}

L'analyse des sonogrammes permet de dresser une carte sédimentologique de synthèse constituée des quatre faciès : vase, sable fin, sable-gravier et roche. Les vases apparaissent le plus souvent sous forme de surfaces très claires, blanches ou légèrement grisées sans structuration apparente (figure la). Les sables fins peuvent le plus souvent être différenciés par les figures sédimentaires de type mégarides qui affectent leur surface (figure $l b$ ). Les sables et graviers sont représentés sur les sonogrammes par des surfaces grises homogènes montrant parfois quelques discrètes mégarides de houle soulignées en général par des dépôts vaseux aux creux des structures. Enfin, les roches se distinguent, généralement, par leur relief accusé et leur aspect irrégulier : elles donnent une image sombre et contrastée (figure la). Leurs directions structurales 
P.-G. SAURIAU et al.
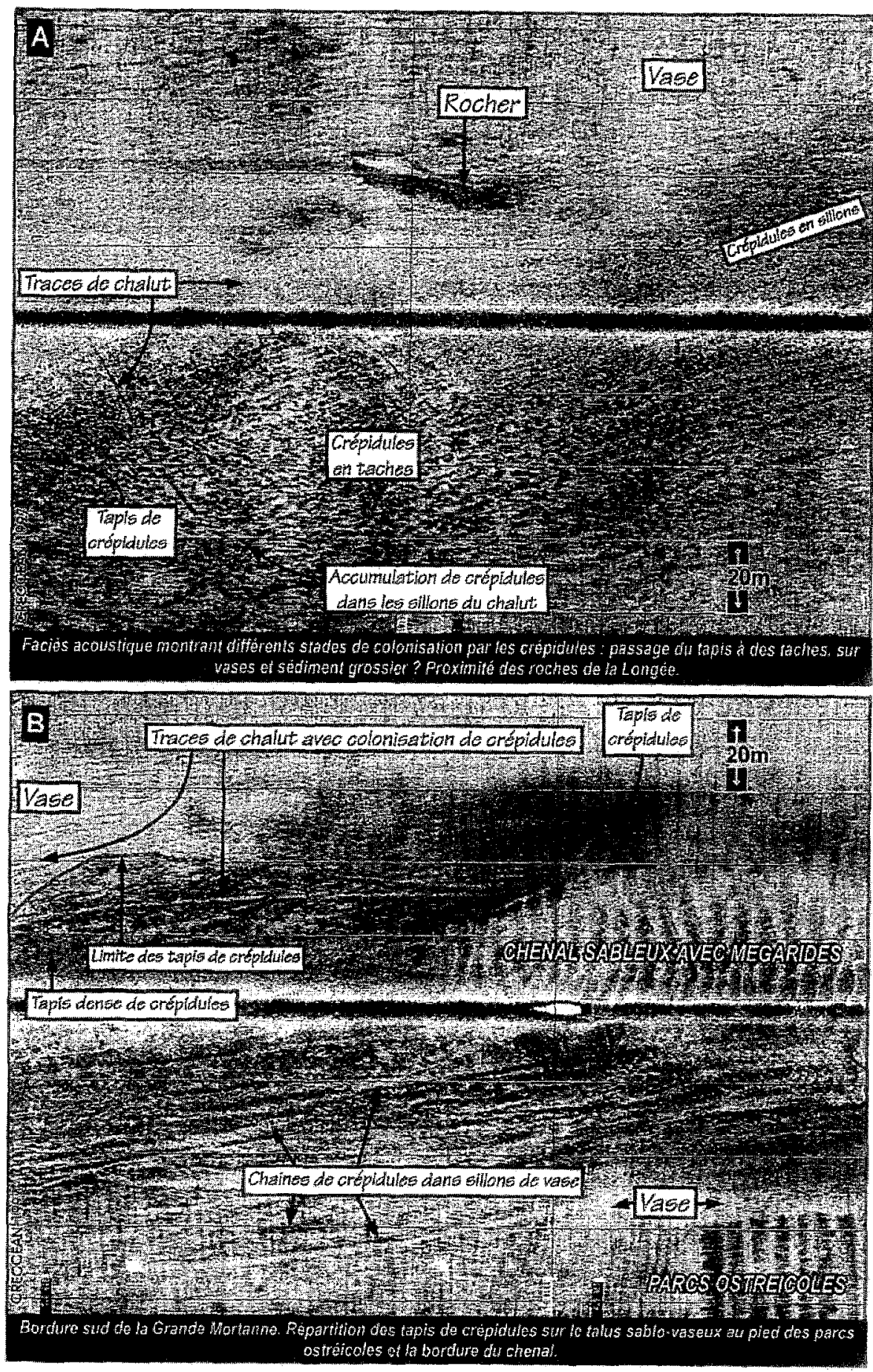

Figure 1. Sonogrammes montrant les 3 stades de colonisation par Crepidula fornicata: tapis, taches et chaŝnes sparses (ici en sillon) sur substrat vaseux et rocheux (A) ou sableux avec présence de mégarides (B).

Figure 1. Side-scan sonar images showing typical colonisation stages of Crepialufornicata: dense ground, colony spot and colony runnel on muddy substrates (A) and sandy substrates with megaripples (B). 
sont par ailleurs très cohérentes avec les observations faites en zones découvrantes: les bancs rocheux subtidaux sont situés en prolongement des affleurements intertidaux marno-calcaires [voir 32,8]. En complément, les traces de chalutage (figures $1 a, 1 b$ ) ainsi que les tables et poches ostréicoles (figure $1 b$ ) apparaissent sans ambiguité sur les sonogrammes et sont d'interprétation aisée.

\subsubsection{Faciès acoustiques en présence de crépidules}

Suivant la terminologie des stades de colonisation fixée par Hamon et Blanchard [14], trois faciès ont été distingués : tapis, taches et chaînes éparses de crépidules.

Les tapis, ou stade ultime de la colonisation, sont formés par des colonies de très fortes densités [ind $\cdot \mathrm{m}^{-2}$ ]. Les images sonar sont sombres et à fort relief (figures $l a, l b$ ). Ce type d'image est parfois représentatif de fonds grossiers avec des galets et graviers en place sur un substrat rocheux tabulaire. La présence de crépidules est cependant fortement suspectée lorsque l'image présente un aspect grumeleux et régulier (figure $1 b$ ). Les moulières naturelles fixées sur un milieu identique peuvent parfois retourner une signature acoustique comparable à celle des colonies de crépidules. L'ambiguité est alors levée grâce aux prélèvements ponctuels. Lorsque les tapis de crépidules sont situés sur des sédiments sablo-vaseux (en fonds clairs sur les enregistrements), l'identification des colonies est beaucoup plus aisée.

Les taches de crépidules sont de surfaces plus limitées et apparaissent sous forme de plages sombres disséminées sur un substrat plus clair. Elles sont parfois organisées en bandes allongées. Elles envahissent préférentiellement les talus en bordure de vasière et les creux des mégarides qui sont formées par les courants sur les fonds sableux (figure la).

Les chaînes éparses de crépidules occupent généralement les zones intensément chalutées et se dispersent dans les sillons laissés par les dragues à pétoncles ou les panneaux de chalut (figures la, $1 b$ ).

\subsection{Cartographie des faciès et gisements de crépidules}

\subsubsection{Répartition des sédiments et dynamique} sédimentaire

Conformément aux cartes bio-sédimentaires levées par Hily [19], les sédiments de vase pure sont, pour l'essentiel, situés sur la bordure orientale de la baie alors que les sables fins ou moyens recouvrent les bancs découvrants et les fonds de chenaux (figure 2). Les sédiments s'enrichissent en graviers et débris coquilliers sur la côte est d'Oléron sans doute par suite de l'action des clapots et des houles qui pénètrent en éventail par le Pertuis d'Antioche au nord-ouest de la baie.

Les mégarides qui apparaissent sur les fonds sableux (figure $1 b$, figure 2) indiquent, par leur pente, un sens de transport des sédiments du sud vers le nord. Ceci révèle une action tractrice prépondérante des courants de jusant, très probablement due à la chenalisation des écoulements lors du retrait de la marée, comme déjà observé sur les fonds de mégarides de la baie de Bristol [16].

\subsubsection{Répartition des gisements de crépidules}

La carte de synthèse du centre de la baie de MarennesOléron met en évidence la précision obtenue sur la localisation des bancs de crépidules (figure 2):

Les tapis de crépidules les plus vastes sont observés en hordure de la vasière de Brouage et dans le prolongement des roches d'Estrée à l'est (Rocher Vert), le long des chenaux de la Grande Mortanne et sur le flanc ouest du banc Dagnas ainsi que sur la bordure nord du rocher Juliar.

Les accumulations de crépidules en taches sont très déve loppées aux deux extrémités nord du banc de Lamouroux et le long de l'ensemble des flancs des chenaux (la Brande à l'ouest; les banes de Charret et de Craze à l'est; la Grande Mortanne, la Courante et le hanc Dagnas au centre de la baie).

Des amas de crépidules sont observés au creux des mégarides de courant, jusqu'au fond des chenaux centraux, mais ce type d'extension reste limité car la mobilité des sables, soumis au jeu des courants de marée, empêche la fixation des chaînes de crépidules. Cependant, la dispersion de colonies en taches, à partir de secteurs rocheux recouverts par un tapis uniforme, vers les vasières subtidales est souvent observable. L'expansion des crépidules se produit alors sous forme de sillons allongés dans le sens général du courant.

\subsection{Estimation quantitative du stock de crépidules}

La cartographie acoustique ne permettant pas de distinguer les crépidules vivantes des crépidules mortes, l'estimation du stock de crépidules a comptabilisé à la fois les coquilles vivantes et les coquilles vides. Le stock total de crépidules (vivantes et mortes) est estimé à $6392 \pm 6460$ 


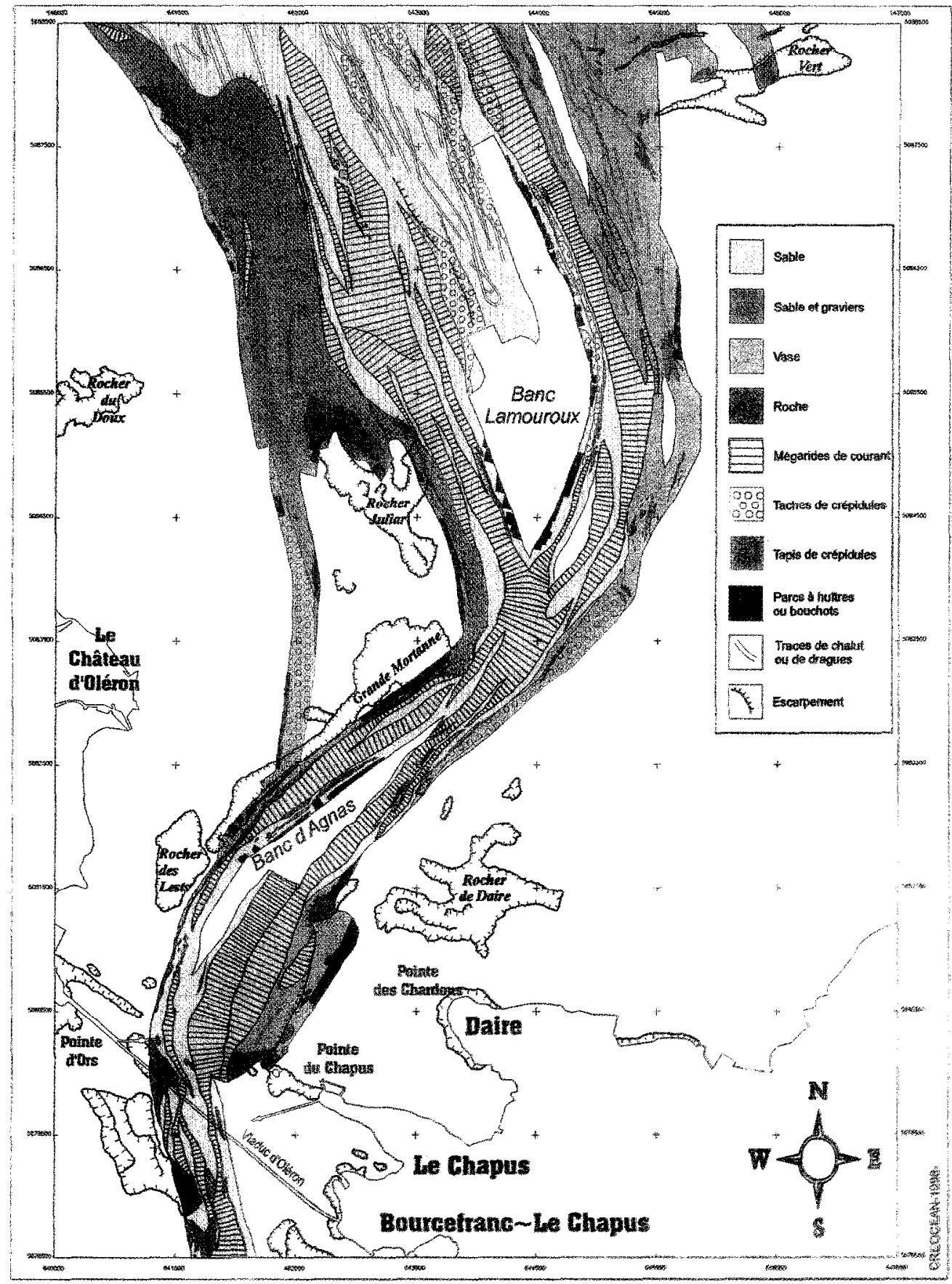

Figure 2. Cartographie des faciès sédimentaires et des zones a crépiduies dans le centre de la baie de Marennes-Oléron. Noter la puésence cies mégarides de courant, des traces de chalutage et des structures conchylicoles (tables à huîtres et bouchots).

Figure 2. Map of the central area of the Marennes-Oléron Bay showing distribution of sediment types and slipper-limpet grounds according to its abundance: fine sand (yellow), coarse sand (orange), mud (yellow-green), rocks (brown), megaripples (blue lines), Crepidula clusters (bluc circlcs), dense Crepidula beds (blue). Bottom-trawl traces (red double line) and shellfish rearing systems (pink) for oysters and mussels are indicated as well as escarpments (purple hatched lines). 
tonnes sur les secteurs de Fouras-Ile d'Aix $(n=18)$ et à $6698 \pm 2431$ tonnes en baie de Marennes-Oléron $(n=62)$. En ne considérant que le stock de coquilles vivantes, celui-ci est de $2494 \pm 3344$ tonnes à Fouras-Ile d'Aix et de $2644 \pm 1137$ tonnes en baie de MarennesOléron. La comparaison du ratio stock/superficie des gisements met en évidence les densités élevées des sites non régulés de la presqu'île de Fouras-île d'Aix (181 ha) par rapport à ceux de Marennes-Oléron (615 ha).

La majorité du stock de la baie de Marennes-Oléron se répartit sur les bancs de Mérignac-Charret, la Courante, Mortanne-Juliar et Longée sud (figure 2). Cette cartographie 1995 reste conforme aux observations de 1981 [26] et de 1984 [30]. Ces quatre bancs sont constitués aux trois quarts de coquilles mortes traduisant très vraisemblablement l'impact des opérations annuelles de nettoyage. Cependant, en 1984, était notée pour la première fois l'apparition du gisement de Lamouroux Est. Son expansion est confirmée en 1995 par l'ampleur du gisement de Lamouroux Nord dont les trois quarts des coquilles sont vivantes (figure 3). La présence concomitante de nombreuses traces de chalutage dans ce secteur très fréquenté (figure 2) suggère une dispersion facilitée par les actions anthropiques, même si le sens de propagation des chaînes de crépidules sur ces fonds sableux suit les mouvements sédimentaires orientés du sud vers lc nord dans ce secteur.

\section{DISCUSSION ET CONCLUSION}

Antérieurement à cette étude, les cartes sédimentaires disponibles en baie de Marennes-Oléron étaient basées sur des grilles de prélèvements discrets avec une nécessaire interpolation des résultats $[19,31]$. Au contraire, une couverture sédimentaire obtenue par imagerie sonar est continue et exhaustive. Elle permet de distinguer les principaux faciès sédimentaires, la contiguïté de leur agencement, les détails de leur morphologie et d'apprécier leur dynamique [3]. La synthèse sédimentologique proposée ici (figure 2) est simplifiée et partielle. Elle est surtout orientée vers la description des faciès à crépidules. Les éléments de dynamique sédimentaire fournis éclairent alors les aspects relatifs à la stabilité des fonds colonisés par la crépidule.

Dans les secteurs charentais prospectés, les chaînes épibenthiques de crépidules se dispersent sur tout type de sédiment depuis les vases, les sables fins, les sables grossiers et les graviers coquilliers jusqu'aux substrats rocheux, ce que notent la plupart des auteurs e.g. [2, 22. 33]. Cette espèce est cependant pratiquement absente dans le sud de la baie de Marennes-Oléron, secteur caractérisé par un fort hydrodynamisme et l'instabilité des fonds formés de dunes hydrauliques. Elle est totalement absente de l'estuaire de la Charente en liaison avec la zone d'influence du bouchon vaseux et la dessalure des eaux [18].

Cette espèce occupe normalement une position bathymétrique subtidale $[33,9]$ même s'il n'est pas rare d'observer de manière éparse des chaînes drossées en milieu intertidal en dehors des parcs ostréicoles. En baie de Marennes-Oléron, la crépidule colonise encore principalement les flancs des chenaux et la frange infralittorale des estrans comme observé sur le banc de Charret [10] et sur la rive nord de la presqu'île de Fouras. La description nouvelle du gisement subtidal de Lamouroux Nord, en regard des cartographies précédentes $[26,30]$ laisse supposer, du fait de l'abondance des traces laissées par les engins traînants (figure 2), une dispersion facilitée des chaînes de crépidules par les activités de chalutage démersal. L'action dispersive reste locale lorsque les crépidules sont transportées par l'engin traînant. Par contre, le rejet des crépidules par dessus bord, lors du tri manuel des captures, peut entraîner une dispersion à plus grande échelle. Cette hypothèse de facilitation de la dispersion par les activités de chalutage est aussi avancée en baie de Saint-Bricuc $[14,15]$. L'extension du gisement de crépidules de Lamouroux Nord est très probablement aussi. liée à la dynamique hydrosédimentaire propre à ce secteur comme l'atteste, de part et d'autre de ce banc, la présence de larges champs de mégarides de sable, générés par les courants de marée.

La prolifération de la crépidule le long du littoral Manche-Atlantique (voir aussi la revue de [13]) a fait l'objet, outrc d'ćtudes de biologie des populations e.g. $[9,21,10]$ de plusieurs estimations de stock dans les années récentes : baie de Saint-Brieuc [14, 15], golfe normanobreton [29], baie de Cancale [Cariguel, 1994 in 4]. Devant l'ampleur des estimations en tonnage cumulé sur ce littoral [voir la synthèse de 4], la question de la régulation des stocks de cette espèce reste toujours récurrente de celle des techniques de traitements à utiliser (rejet à terre, rejet en mer après traitement et/ou valorisation) comme déjà analysé dans les années 1920 par [12]. Cependant, la comparaison des deux cartographies quantitatives des gisements de crépidules réalisées dans la baie de Marennes-Oléron à onze ans d'intervalle (1984 et 1995) permet, au vu de la similarité des estimations de 


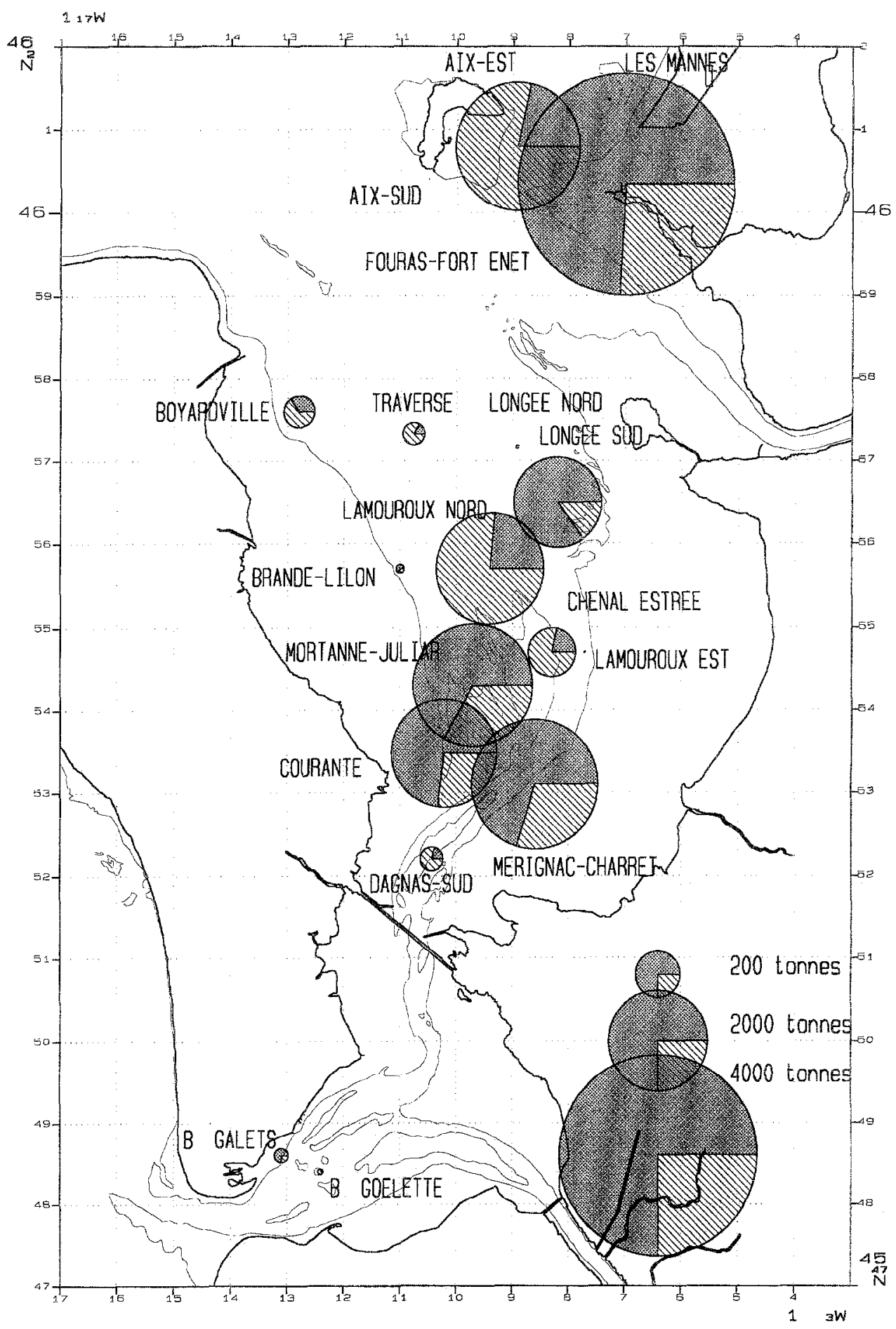

Figure 3. Répartition géographique des stocks (tonnes) de crépiđules sur les gisements de la presqu'île de Fouras, l'île d'Aix et en baie de Marennes-Oléron avec distinction des fractions coquilles vivantes (hachure) et coquilles mortes (trame foncée).

Figure 3. Map of Crepidula formicata grounds (stocks in tons) within the Fouras-Aix area and the Marennes-Oléron Bay. The ratio between live chains (line) and dead chains (shadded frame) is indicated. 
stock, de conclure à l'efficacité des dragages de nettoyage mis en oeuvre depuis quinze ans dans cette baie. I es tonnages annuels pêchés, en moyenne de 1000 à 1500 tonnes de coquilles depuis 1990 , ont vraisemblablement permis de stabiliser la progression du stock des principaux gisements de crépidules de la baie. En revanche, la propagation des chaînes de crépidules dans la baie reste sous l'influence de facteurs naturels (courant et charriage) et d'actions anthropiques (chalutage et rejet des captures non commerciales) qui agissent à différentes échelles spatiales.

\section{Remerciements}

Les autreurs remercient particulièrement l'équipage du catamaran Les Deux Mouettes ainsi que les patrons des chalutiers Maxi II et Rainbow Warrior pour leur aide précieuse lors des opérations à la mer. Ils remercient aussi D. Hamon pour ses conseils à propos de l'analyse bio-sédimentaire des enregistrements sonar. Ce travail a été financé par le Conseil Général de la Charente-Maritime (contrats $n^{\circ} 42142$ et 502869), l'IFREMER (contrat $\mathrm{n}^{\circ}$ 95-5-545001) et le CNRS.

\section{RÉFÉRENCES}

[1] Andrews J.E., Craig J.D., Hardy W.A., Investigations of the deep-sea floor by side-scan sonar techniques, Deep-Sea Res. 24 (1977) 975-985.

[2] Barnes R.S.K., Coughlan J., Holmes N.J., A preliminary survey of the macroscopic bottom fauna of the Solent, with particular reference to Crepidula fornicata and Ostrea edulis, Proc. Malac. Soc. Lond. 40 (1973) 253-275.

[3] Berné S., Augustin J.M., Braud F., Chene G., Walker P., Cartographie et interprétation de la dynamique sédimentaire des plates-formes continentales : améliorations de la technique d'observation par sonar latéral, Bull. Soc. Géol. Kr. 2 (1986) $437-446$.

14」 Blanchard M., Origine et état de la population de Crepidula fornicata (Gastropoda Prosobranchia) sur le littoral français, Haliotis 24 (1995) 75-86

[5] Cochran W.G., Sampling Techniques, 3rd ed., Wiley and Sons, New York, 1977.

[6] Cole H.A., Hancock D.A., Progress in oyster research in Britain 1949-1954, with special reference to the control of pests and diseases, Cons. Int. Explor. Mer. Rapp. et Proc.-Verb. 140 (1956) 24-29.

[7] Cooper J.E., Note on Crepidula fornicata, J. Conch. 11 (1905) $227-228$.

[8] Corlieux M., Étude géologique abrégée de la Charente-Maritime, Ann. Soc. Sci. Nat. Charente-Marit. Suppl., 5 (1972).

[9] Coum A., La population de crépidules Crepidula fornicata (L., 1758 ) en rade de Brest : écologie et dynamique, Thèse $3^{\text {ème }}$ cycle, Université Bretagne Occidentale, Brest, 1979, 146 p.

[10] Deslous-Paoli J.M., Crepidula fornicata L. (gastéropode) dans le bassin de Marennes-Oléron : structure, dynamique et production d'une population, Oceanol. Acta 8 (1985) 453-460.

[11] Deslous-Paoli J.M., Héral M., Crepidula formicata L. (Gastéropode, Calyptracidae) dans le bassin de Marennes-Oléron : composition et valeur énergétique des individus et des pontes, Oceanol. Acta 9 (1986) 305-311.
[12] Dollfus R.Ph., Résumé de nos principales connaissances pratiques sur les maladies et les ennemis de l'huître, Notes et Mém., Off. Scient, Tech. Pêches Marit. 7 (1921).

[13] Gruet Y., Baudet J., Les introductions d'espèces d'invertébrés marins, in : Dauvin J.C. (éd.), Les biocénoses marines et littorales françaises des côtes Atlantique, Manche et Mer du Nord. Synthèse, menaces et perspectives, MNHN, Paris, 1997, pp. 242-250.

[14] Hamon D., Blanchard M., Etat de la prolifération de la crépidule (Crepidula fornicata) en baie de Saint-Brieuc, Rap. IFREMER/DEL, 94.14 (1994).

[15] Hamon D., Peuplements benthiques des fonds meubles. Distribution de la faune benthique, distribution de la crépidule (Crepidula fornicata), in : Augris C., Hamon D. (éd.), Atlas thématique de l'environnement marin en baie de Saint-Brieuc (Côtes d'Armor), IFREMER, Brest, (1996) 45-51.

[16] Harris P.T., Collins M.B., Side-scan sonar investigation into tcmporal variation in sand wave morphology: Helwick sands, Bristol Channel, Geo-Mar. Lett. 4 (1984) 91-97.

[17] Héral M., Deslons-Paoli J.M., Bacher C., La capacité biotique des bassins ostréicoles, in : Troadec J.P. (éd.), L'homme et les ressources halieutiques, IFREMER, Plouzané, 1989, 225-259.

[18] Héral M., Razet D., Deslous-Paoli J.M., Manaud F., Truquet I., Garnier J., Hydrobiologie du bassin de Marennes-Oléron. Résultats du réseau national d'observation : 1977 à 1981, Ann. Soc. Sci. Nat. Charente-Maritime 7 (1984) 259-277.

[19] Hily C., Écologie benthique des Pertuis charentais. Thèse $3^{\text {ème }}$ cycle, Université Bretagne Occidentale, Brest, 1976, 236 p.

[20] Korringa P., Crepidula formicata as an oyster-pest, Cons. Int. Explor. Mer. Rapp. et Proc.-Verb. 128 (1951) 55-59.

[21] Le Gall P., Étude expérimentale de l'association en chaîne et de son influence sur la croissance et la sexualité chez la crépidule Crepidula fornicata Linné 1758 (mollusque mésogastéropode). Thèse Doct. es Sci. Nat., Univ. Caen, 1980, 251 p.

[22] Loomis S.H., Van Nieuwenhuyze W., Sediment correlates to density of Crepidula fornicata Linnaeus in the Pataguanset River, Connecticut, The Veliger 27 (1985) 266-272. 
[23] Lubet $8 .$, Le Gall P., Recherches préliminaires sur la structure des populations de Crepidula fornicata Philb., mollusque mésogastéropode, Bull. Soc. Zool. Fr. 97 (1972) $211-222$.

[24] Marteil L., La crépidule (Crepidula fomicata L.) en France, Sciences et Pêche, Bull. Info. Doc. Inst. Pêches Marit., 121 (1963) $1-6$.

[25] Marteil L., Extension de l'aire géographique de Crepidula fornicata I. pendant 1'année 1964, Sciences et Pêche, Bull. Info. Doc. Inst. Pêches Marit. 135 (1965) 5-6.

[26] Massé H., Lagardère J.P., Recherches écologiques sur un écosystème estuarien à vocation aquicole, le bassin de MarennesOléron, Rap. CNEXO, 81/2503 (1981) 43-142.

[27] Murie J., 'Slipper limpet' or 'boat shell' (Crepidula fornicata): its introduction and influence on Kent and Essex oyster-beds, Zoologist 15 (1911) 401-415.
[28] Prior D.B., Coleman J.M., Roberts H.H., Mapping with side scan sonar, Offshore 41 (1981) $15 !-16 !$.

[29] Quiniou F., Blanchard M., État de la prolifération de la crépidule (Crepidula fornicata $L_{4}$ ) dans le secteur de Granville (golfe normano-breton), Haliotis 16 (1987) 513-526.

[30] Sauriau P.G., Les nollusques non cultivés du bassin de Mărennes-Oléron : quantification et répartition géographique des stocks, Haliotis 16 (1987) 527--541.

[31] Sauriau P.G., Mouret M., Rincé J.P., Organisation trophique de la malacofaune benthique non cultivée du bassin de MarennesOléron, Oceanol. Acta 12 (1989) 193-204.

[32] Verger F., Marais et wadden du littoral français ; étude de géomorphologie, Biscaye Frères, Bordeaux, 1968.

[33] Walne P.R., The biology and distribution of the Slipper Limpet Crepidula fornicata in Esscx rivers, Fish. Invest. 2 (20) (1956). 